\section{Cahiers de Narratologie}

Analyse et théorie narratives

$35 \mid 2019$

Le style comme événement

\title{
Événementialité du style et force émergentielle de la microsyntaxe
}

Nicolas Laurent

\section{(2) OpenEdition}

1 Journals

\section{Electronic version}

URL: http://journals.openedition.org/narratologie/9342

DOI: 10.4000/narratologie.9342

ISSN: 1765-307X

\section{Publisher}

LIRCES

\section{Electronic reference}

Nicolas Laurent, "Événementialité du style et force émergentielle de la microsyntaxe », Cahiers de Narratologie [Online], 35 | 2019, Online since 03 September 2019, connection on 05 September 2019. URL : http://journals.openedition.org/narratologie/9342; DOI : 10.4000/narratologie.9342

This text was automatically generated on 5 September 2019

Article L.111-1 du Code de la propriété intellectuelle. 


\title{
Événementialité du style et force émergentielle de la microsyntaxe
}

\author{
Nicolas Laurent
}

La vitalité contemporaine de la notion de style, qu'on rencontre aussi bien, par exemple, dans les analyses réflexives des styles d'existence et des pratiques de vie que dans des discours non savants, où elle permet, selon un usage plus simplement "régulateur " (Martin, 1994:1311), d'épingler telle ou telle différenciation, met en relief le fait que «le style apparaît aujourd'hui "en acte", comme l'instrument d'un faire, d'une pratique », et qu'il est donc devenu « pertinen[t] dans tous les domaines de l'agir » (Jenny, $2011: 12$ ). L'« extension du domaine du style » (Macé, 2010) montre exemplairement que se loge, au cœur de la notion, une certaine opérativité, et que s'impose en effet une "pragmatique du style ", pour reprendre le sous-titre du collectif dirigé par L. Jenny (2011).

2 Toutefois, cette extension du style demande, pour nous, stylisticiens, non de l'existence, mais du texte, à être placée au cœur même de l'invention littéraire. De fait, le style - en lequel il convient de ne pas voir une entité statique mais quelque chose qui se constitue " en acte ", dans l'œuvre - gagne à être envisagé dans sa relation avec la notion d' événement. S'interroger sur le style, dans ce cadre, invite à considérer l'événementialité des formes, leur manière d'apparaitre et de signifier en contexte. On verra que la notion d'émergence, entendue ici lato sensu, permet de modéliser des interrelations complexes dont le style est le lieu, ou le lien, invitant, à réception, à parcourir l'entier du continuum discursif. On s'attachera en particulier à montrer l'importance - quelque peu sousestimée dans la stylistique contemporaine - des morphèmes et des traits grammaticaux, dont le sens, instructionnel ou pré-conceptuel, peut être mobilisé conceptuellement et donner lieu à des effets de figuration et d'implication prédicative. Le point de vue développé ici sera celui d'un nécessaire ancrage référentiel du style, et donc d'une mise en tension, à travers lui, de la langue et du «monde », formant ce qu'on peut appeler un événement de représentation. 


\section{Du style comme événement}

3 Qu'est-ce qu'un événement? En une première approche, comme l'indique le philosophe S. Vinolo, on peut penser que l'événement, à la différence « des changements ordinaires qui ont [aussi] lieu dans le monde », « arrive » comme « en exception » dans ce monde, en ce qu'il est irréductible au simple jeu de «variations » qui commandent le devenir de celui-ci : l'événement n'est pas une simple "modification [...] banale [...] de l'être-là » mais bien, finalement, une « modification $d u$ monde » lui-même (Vinolo, 2013:52 et 57).

Encore faut-il disposer de critères permettant de distinguer entre événement et « fait non-événementiel». On sait qu'A. Badiou a développé une théorie ontologique de l'événement: selon lui, un événement se caractérise par sa "réflexivité " (étant en « exception dans ce qui apparaît », il «s'appartient à lui-même» (2009: 89 et 94)), son « intensité (il existe maximalement) » et sa " puissance » : modification du monde, il fait exister ce qui était jusque-là inexistant dans le monde (2009 : 94-95). Ce dernier point est relié à la thèse fondamentale selon laquelle, dans sa pure contingence, l'événement donne lieu, paradoxalement, à une vérité universelle. Dans ce cadre, la vérité est un processus (« les vérités [...] ne font pas qu'être, elles apparaissent », $2006: 18$ ), et selon un mode qui n'est pas celui de la descente (d'un Ciel des Idées) ou de l'incarnation, mais du "déploiement » (2009: 99) à partir de l'événement. Autrement dit, tout surgissement événementiel se signale réflexivement, et intensément, dans l'immanence d'un monde et comme point de départ - vite évanoui - d'un processus de vérité.

La théorie d'A. Badiou a le mérite de nouer une « logique de l'apparaitre » à un processus de vérité qui se développe dans le monde en étant « en exception » dans ce monde. Elle invite, pour ce qui nous concerne, à envisager le style comme ce qui participe d'une procédure de vérité artistique - c'est-à-dire d'une "nouvelle capacité à informer le sensible », d'« un élargissement en devenir de la mise en forme » (2006:82) - et donc comme ce qui relève de l'universel. Cet universel, insistons sur ce point, n'est pas l'incarnation sensible d'une Idée transcendante séparée, comme le rappelle A. Badiou qui, au début de Logiques des mondes, prend l'exemple du motif du cheval peint par le Maître de la Grotte Chauvet : cette image, en tant que "vérité éternelle ", " est non pas du tout la descente de l'Idée dans le sensible, mais la création sensible de l'Idée » (2006:27).

La dimension d'événement de l'œuvre - et donc du style - tient à une disposition nouvelle dans l'art de lier «la part du sensible et celle de la pensée » (2018: 544) et, plus précisément, au fait que l'œuvre opère « un déplacement [...] de la différence entre forme et informe » et est donc "chargée d'un nouveau concept de la forme »: celui-ci l'inscrit "dans l'histoire des vérités artistiques» (2018: 544). Sur ce point, il faut signaler que, si le contexte historique rend possible telle disposition nouvelle dans l'art de lier forme et pensée, il ne l'explique pas, tant il est vrai que toute œuvre, en tant qu'œuvre, est irréductible aux causes qu'on peut invoquer pour en rendre compte. Selon S. Vinolo,

l'événement n'est [...] pas provoqué par des causes que nous trouverions dans le monde pré-événementiel, causes que nous devrions pouvoir reproduire. L'événement se suffit à lui-même, il surgit en lui-même, à partir de lui-même. (Vinolo, 2013 : 55)

7 Il faut sans doute relativiser la dernière phrase, dans la mesure où la réflexivité de l'événement est bien située dans un contexte - et où, à réception, la connaissance de ce contexte augmente l'acuité cognitive et donc la sensibilité à l'innovation formelle - mais 
il est certain que le style - puisque c'est de cela qu'il est question - n'est pas entièrement déductible de ce que l'on sait du «monde pré-événementiel» (le contexte historique, l'auteur, la société, etc.). Quelque chose en plus, pour ainsi dire, a eu lieu - et a encore lieu dans l'expérience qu'en fait le lecteur, malgré - ou grâce à - la distance synchronique (l'œuvre est en exception dans le monde) et diachronique (la « communication différée » qu'elle représente en appelle virtuellement à la postérité). Que le lecteur se rassure ici : il n'y a, de notre part, nul désir d'idéaliser les oeuvres, mais simplement le souhait de rendre compte de ce que certaines œuvres, pour ainsi dire, ne sont pas seulement faites mais font quelque chose, et continuent donc d'agir sur nous et de se présenter comme des événements.

8 C'est ici qu'il faut distinguer, avec L. Jenny, deux manières opposées d'appréhender le style, comme "enchâssement de caractérisations ", somme de propriétés extrinsèques indiquant des «appartenance[s]» (2000: 102) - au genre du théâtre, à une manière historiquement marquée d'utiliser tel type de vocabulaire, etc. -, ou comme dynamique de construction de formes nouvelles porteuses de sens, dont la « logique interne » (2000: 103) n'est pas réductible à une combinaison de propriétés extrinsèques. Ces deux conceptions renvoient à deux voies d'accès à l'individu. Le philosophe $\mathrm{S}$. Chauvier indique ainsi qu'on peut penser à un individu soit comme «à une entité numérique d'une espèce ou d'une sorte données » - ce qu'il appelle une « hénade »-, soit comme à " un absolu singulier » - ce qu'il appelle une « monade » $(2010: 3)$. Il précise son point de vue :

La situation est analogue à celle d'un sujet photographique qu'on photographierait tantôt sans zoom, tantôt avec un zoom macro. Dans le premier cas, le sujet se détache sur un fond plus ou moins net et peut être entouré de divers autres sujets photographiques. Dans le second cas, le sujet occupe seul la scène, il est son propre fond et on le voit moins lui qu'on ne voit des choses de lui ou en lui. (2010:3-4)

La «manière dominante » d'accéder cognitivement à un individu, nous dit-il, c'est celle qui y voit une « hénade». Pourtant, se former un concept singulier de Pierre implique de voir dans Pierre plus qu'un particulier instanciant le concept de « homme », de « voisin » et / ou de «clarinettiste »... Dans le champ des études stylistiques, les deux approches, catégorisante (discursive, rhétorique) et singularisante, sont évidemment complémentaires, mais le «zoom» de la stylistique est bien un «zoom macro», parce qu'en tant que discours individué, et appelant, à des degrés divers, une attitude esthétique qui valorise sa "réalité propre" (Schaeffer, 2011: 146), l'œuvre n'est pas seulement une entité "hénadique ", elle est aussi une entité «monadique »: il existe entre le discours et l'œuvre la même différence logique qui sépare le particulier de l'individu.

Comment, dans ce cadre, rendre compte de l'opérativité individuante du style? Tout indique que la théorie de l'émergence, corrélée à une épistémologie de la complexité, peut être d'une grande utilité. I. Yocaris, dans Style et semiosis littéraire, a déjà proposé d'utiliser la théorie de l'émergence pour rendre compte de certains faits de style :

les faits de style (à commencer par ceux qui reposent sur des relations latérales²) peuvent avantageusement être considérés comme des propriétés émergentes d'un système holistique, englobant selon les cas le lecteur et le (para/inter)texte ou bien le lecteur, le (para/inter)texte et l'entour pragmatique. $\left(2016: 59^{3}\right)$

11 La notion d'émergence sera définie ici à partir des analyses qu'en a faites E. Morin dans $L a$ Nature de la nature, ouvrage consacré aux sciences de la nature mais promouvant plus généralement, à l'ouverture de la grande aventure de La Méthode, un principe de complexité destiné à refonder une nouvelle manière d'envisager les sciences de l'homme ${ }^{4}$. Cette notion intervient crucialement dans une réflexion sur la nature organisationnelle 
du système, « unitas multiplex ", à la fois « un et homogène » - puisque ce système est un tout - et "divers et hétérogène » - puisqu'il est "constitué de parties diverses » mais « interrelationnées » $(1977: 105)$. Elle permet de comprendre pourquoi «le tout est plus que la somme des parties » :

Le système possède quelque chose de plus que ses composants considérés de façon isolée ou juxtaposée :

- son organisation,

- l'unité globale elle-même (le « tout »)

- les qualités et propriétés nouvelles émergeant de l'organisation et de l'unité globale. (1977: 106)

Les émergences sont «les qualités ou propriétés d'un système qui présentent un caractère de nouveauté par rapport aux qualités ou propriétés des composants considérés isolément ou agencés différemment dans un autre type de système " (1977: 106). Elles "[ont] donc vertu d'événement», étant des "qualité[s] qui ne se laisse[nt] pas décomposer, et que l'on ne peut déduire des éléments antérieurs » (1977 : 108). Elles sont présentes, par exemple, dans l'organisation de l'atome, mais elles expliquent aussi, selon E. Morin, «les notions apparemment élémentaires que sont matière, vie, sens, humanité ", qui « correspondent en fait à des qualités émergentes de systèmes » (1977 : 107). Les émergences caractérisent le tout, mais aussi les parties, puisque ces parties peuvent acquérir, au sein du tout, des qualités qu'elles n'auraient pas isolément : « dès lors, non seulement le tout est plus que la somme des parties, c'est la partie qui est, dans et par le tout, plus que la partie » $(1977: 108)$.

13 C'est dans cette interrelation complexe des parties et du tout que peut être localisée l'événementialité du style, qualité émergente globale, mais aussi organisation significative faisant de la partie " plus que la partie » en la faisant participer au et du tout. En quelque sorte, le style multiplie, à tous les paliers de la phrase et du texte (mot, syntagme, proposition, etc.), les points d'application d'un principe de récursivité, ce terme devant être entendu dans un sens non pas strictement linguistique, mais plus largement organisationnel. Selon E. Morin,

l'interrelation qui lie l'explication des parties à celle du tout et réciproquement est en fait invitation à une description et explication récursive: la description (explication) des parties dépend de celle du tout qui dépend de celle des parties (1977: 125)

Encore doit-on entendre ici la notion d'émergence dans un sens épistémologiquement affaibli : l'hypothèse qui s'impose dès lors qu'on s'attache précisément au texte littéraire, c'est moins celle d'une émergence ou d'un ensemble d'émergences « surgi[ssant] de façon discontinue » et comme « irréductible[ment] » au sein du contexte (Morin, 1977 : 108-109) que celle d'une densification formelle et sémantique, appelant au contraire une analyse en continuum des «interrelations » des parties et du tout ${ }^{5}$. Cette densification présuppose la possibilité, bien analysée par G.-G. Granger, d'une «superposition des codes » et d'un « codage a posteriori » allant " au-delà » de la structuration « a priori » (1988: 191). Les émergences représentent, selon nous, des types particuliers de structurations sémantiques a posteriori motivant la mise en relation d'éléments linguistiques ne relevant pas nécessairement du même palier textuel. Ce «surcodage» est au principe de l'individuation discursive :

l'objet s'individue lorsque plusieurs structurations concurrentes sont simultanément possibles, - et non pas seulement des structurations de plus en plus fines et pour ainsi dire emboîtées, mais des structurations chevauchantes, superposées, certaines partielles, d'autres globales pour un objet donné (1988 : 203). 
Il faut, en conséquence, accorder toute son importance au concept d'œuvre, qui implique "l'intégra[tion de] sa propre clôture ", " cette clôture immanente [étant] le propre de toute œuvre relevant de la procédure de vérité artistique » (Badiou, 2018: 541). Précisons cependant que cette clôture doit plutôt être pensée comme une "autonomie relative " (Morin, 1977: 143), puisqu'il est certain que «l'observateur fait aussi partie de la définition du système observé » et que « le système observé fait aussi partie de l'intellect et de la culture de l'observateur-système " (Morin, 1977 : 143). L'œuvre est ainsi, comme le rappelle J.-M. Schaeffer, "ce qu'elle devient à travers ses multiples réactivations " combinant propriétés «native(s)» et «concrétisations historiques» (2011: 141). En conséquence, l'événementialité du style conjoint naturellement une "réalité propre " (Schaeffer, 2011: 146) - elle est « réellement » située dans les appariements des formes et des sens - et une appropriation vivante - elle est non moins « réellement » reçue par le lecteur.

\section{« Zoom macro » et microsyntaxe}

Les remarques précédentes le suggèrent : le style envisagé comme processus « implique » en fait une pluralité, "un ensemble de processus» (Herschberg-Pierrot, 2005: 34), et l'événementialité du style associe donc, combine, fusionne... des interrelations qui sont autant de micro-événements formels et sémantiques ${ }^{6}$. Dans ces faits de (sur) structuration où l'entier du texte est potentiellement sollicité, les morphèmes grammaticaux, qui « met[tent] en jeu », par la nature de leur sens, plus instructionnel que conceptuel, « des mécanismes dynamiques » (Kleiber, 1999 : 50), jouent un rôle essentiel. Il n'est pas rare qu'un simple mot grammatical concentre réflexivement une "vision ", une « représentation » singulières - ce qui ne signifie pas qu'il est la simple synthèse de ce qui est dit mais que les tensions, nées de ses relations avec son entour, opèrent un « déplacement ", pour reprendre le terme d'A. Badiou, «de la différence entre forme et informe » et renouvellent donc le rapport existant entre forme et contenu. Le "figural » de L. Jenny est par exemple illustré par un usage singulier, dans une traduction du Tao-töking, de la préposition en :

Soit donc un exemple d'apparition du figural. Tel matin, je feuillette en dilettante une traduction savante du Tao-tö-king. Lecture distraite, lointaine et peu sérieuse jusqu'à ce que je trébuche sur ces mots :

l'ascension d'une terrasse en printemps (1990: 20)

Jenny se rend compte que «c'est ce en (printemps) qui [1'] a arrêté par son équivoque " (1990: 21) et l'analyse réflexive de cet «événement local que constitue l'expression terrrasse en printemps " montre que la préposition en, par opposition à à qui introduirait une « indication [...] temporelle [...]», traite le printemps comme une apparence typique, sur le modèle d'un "arbre en fleurs». Ainsi, le figural "représente (imitativement) quelque chose du monde en re-présentant (en présentant à neuf) la forme de la langue » (1990 : 25-26).

18 Comme le montre l'exemple de L. Jenny, une interrelation inédite peut localiser sur un mot grammatical quelque chose qui est de l'ordre de l'événement: une "création sensible » qui " présent[e] à neuf» la puissance de représentation de la langue. Cette événementialité a pour signe, à réception, le dépli conceptuel, et même prédicatif (c'està-dire une articulation en termes de sujet et de prédicat), du morphème : 
19 (i) son sens est l'objet d'une pensée du possible, c'est-à-dire d'une pensée conceptuelle ${ }^{7}$ (le sens de en se différencie d'autres sens possibles de en et en se distingue, in absentia, de la préposition $\grave{a}^{8}$ ), et c'est dans cette mesure que le fait de style, en effet, nous renvoie à la langue, système des possibles ;

(ii) si cet arrêt de la pensée conceptuelle se produit sur en, c'est parce que la réflexivité de son "apparaître ", son " effet de présence ", se combine à un "effet de signification » contextuel. Nous reprenons ces deux termes à H. U. Gumbrecht selon qui la mise en tension de ces deux « effets » est propre aux arts :

je dirais que le trait spécifique qu'il [Luhmann] a relevé dans le système de l'art est peut-être bien la possibilité de vivre (erleben) en simultané des effets de présence et des effets de signification. Chaque fois qu'elle se présente, cette simultanéité peut être vécue comme une oscillation ou une tension. Ce qu'il faut retenir, c'est que, au sein de cette constellation spécifique, la signification ne mettra pas entre parenthèses les effets de présence, ne les fera pas disparaître, et que la présence physique - sans mise entre parenthèses - des choses (d'un texte, d'une voix, d'une toile recouverte de couleurs ou du jeu d'une équipe) ne réprimera pas en définitive la dimension signification. (2010:165)

Le mot en sur lequel l'événement est localisé implique en quelque sorte un contenu prédicatif qui, tout en étant en rapport avec la prédication expliquée, ne s'y réduit pas. La "partie» interrelationnée avec le «tout» s'augmente significativement, mais tensionnellement, et l'effet est d'autant plus remarquable qu'il sollicite maximalement un monosyllabe, une simple préposition.

Le contexte intervient à des degrés divers dans la "transformation " sémantique des mots grammaticaux. Dans l'exemple de L. Jenny, qui illustre un cas de «trope prépositionnel» $(1990: 24)$, c'est principalement la mise en relation, qui fait localement écart, entre la préposition en et son complément syntaxique qui déclenche le « figural». D'autres contextes peuvent paraître plus contraignants, notamment quand une occurrence se présente comme une variante in praesentia. Soit, par exemple, cet autre emploi de la préposition en dans les Pensées de Pascal :

Les stoïques disent : «Rentrez au-dedans de vous-même. C'est là où vous trouverez votre repos. » - Et cela n'est pas vrai.

Les autres disent : "Sortez dehors et cherchez le bonheur en un divertissement.» Et cela n'est pas vrai. Les maladies viennent.

Le bonheur n'est ni hors de nous ni dans nous. Il est en Dieu, et hors et dans nous. ( Pensées, éd. P. Sellier, Paris, Classiques Garnier, 2011, p. 166)

Le double effet de "présence » et de "signification » sensible dans "Il est en Dieu » s'explique pour partie par l'importance contextuelle d'un paradigme prépositionnel actualisé. La préposition en vient transcender l'antonymie existant entre les prépositions plus colorées «hors (de)» et «dans $»^{10}$ et figure le mouvement - rendu possible par Dieu - grâce auquel les antinomies et les contrariétés humaines peuvent paradoxalement coexister et même être dépassées. En conséquence, elle constitue le point de résolution grammatical de l'antéisagoge ${ }^{11}$, et son énonciation coïncide avec l'inversion du signe de la polysyndète, puisque celle-ci, d'abord négative ( « ni hors de nous ni dans nous »), devient positive (« et hors et dans nous »). À la différence de l'occurrence analysée par L. Jenny, en fait ici nécessairement l'objet d'une focalisation contrastive puisque le mot participe crucialement de la dialectique des Pensées, qui s'incarne dans la grammaire. Mais si l' explication paraît plus contrainte par ces variations structurales, cet en-là n'en est pas moins le support d'un événement représentationnel où se rejoue, là encore, le rapport entre forme et pensée. Non seulement le contexte étend la capacité à dire du morphème 
- ce qui, du côté du «monde ", implique une extension de la dicibilité de celui-ci -, mais encore la forme elle-même, en dernier ressort, n'est pas stabilisée : en est mis en relation avec les prépositions, mais aussi avec la double polysyndète, le jeu sur le signe de l'énoncé (négatif puis positif), la forme typique de l'antéisagoge, l'épitrochasme, etc. La multiplication des appariements formels et sémantiques, fondés sur une logique de motivation, produit, à réception, autour de cette expression pourtant courante, «en Dieu », un effet d'événement et de « création sensible ».

Un autre exemple pris dans les Pensées de Pascal valorise potentiellement, non un morphème grammatical libre, mais une simple flexion verbale :

Toutes ces contrariétés qui semblaient le plus m'éloigner de la connaissance d'une religion est ce qui m'a le plus tôt conduit à la véritable. (Pensées, éd. P. Sellier, Paris, Classiques Garnier, 2011, p. 165).

Ce fragment signifie, selon la paraphrase proposée par le site Internet consacré aux Pensées de Pascal, qu'

alors que les « contrariétés » de la nature humaine peuvent conduire au scepticisme le plus complet, elles conduisent au contraire à la vraie religion, qui est seule capable d'en faire tenir ensemble les aspects contraires en apparence ${ }^{12}$.

Toutefois, pour formuler son paradoxe, Pascal a éliminé toute indication d'opposition et recouru à une phrase attributive qui pose, au contraire, une coréférence stricte entre un sujet pluriel, le $\mathrm{SN}$ «toutes ces contrariétés qui... », et un attribut neutre, singulier, la relative périphrastique "ce qui m'a le plus tôt...». La flexion du verbe être peut surprendre le lecteur moderne : la tête du SV est au singulier alors que le prédicat se rapporte à un sujet pluriel. On peut rappeler que «les phrases à verbe être sont dans l'usage classique une forte zone de variation de l'accord» et qu'en l'occurrence, «le singulier du verbe est l'indice d'une vision collective et non pluralisante du référent " (Fournier, 1998: 30). Va dans le sens de cette analyse la présence, dans le SN sujet, du prédéterminant "toutes", qui rassemble les occurrences dans un tout, et ce SN signifierait donc, comme le suggère $\mathrm{N}$. Fournier, qui relève la phrase de Pascal, « l'ensemble de ces contrariétés » $(1998: 30)$. Une seconde hypothèse, toutefois, peut être envisagée, qui ne normalise pas l'accord, mais en creuse la puissance de figuration en contexte. Car l'accord au singulier met en tension "présence " et "signification", réflexivité de la forme et visée référentielle - et la syllepse de nombre accède au statut de « vraie » figure. En effet, le singulier « est » concentre la force du paradoxe pascalien, selon lequel seule la vérité chrétienne peut transcender la diversité des " contrariétés ». Il est remarquable que le verbe serve de pivot à une série d'antithèses grammaticales et sémantico-référentielles - déterminant démonstratif pluriel ("ces (contrariétés)») vs pronom démonstratif singulier («ce (qui)»), article indéfini à valeur générique (" une (religion)») vs article défini à valeur spécifique («la (véritable)»)... : ce qui précède le verbe vise la pluralité, externe ou interne, ce qui le suit l'unité (« ce qui ») et l'unicité ( «la véritable »). Ajoutons que le contenu, qui pourrait donner lieu à une maxime paradoxale, est élaboré sous la forme d'une courte phrase narrative au passé qui traduit également un embrayage temporel, et qu'à l'imparfait succède, après "est», le passé composé, construit à partir du présent: l'unicité incommensurable de la vérité chrétienne est présentée à travers "est». Autrement dit, l'accord au singulier, rendu possible par la grammaire du XVII e siècle, est rendu nécessaire par la « vision» de Pascal. La flexion du verbe être devient porteuse de la dialectique des Pensées et cette signification, purement locale, représente une émergence spécifique, invitant à reconsidérer récursivement la totalité de la phrase dans laquelle elle prend place. Dans ce parcours, le sens 
instructionnel ou pré-conceptuel de la flexion du verbe est, là encore, mobilisé conceptuellement et entre, au même titre que les éléments plus immédiatement descriptifs, dans la prédication opérée par la phrase. Bref, c'est un micro-événement morphosémantique par lequel, par le biais d'une structuration stylistique dense, « apparaît » réflexivement, et dynamiquement, la logique des Pensées.

Naturellement, la normalisation grammaticale, qui consiste à voir dans « est » un fait d'accord typique de la langue du XVII ${ }^{e}$ siècle, reste toujours possible et, en ce sens, c'est bel et bien le récepteur qui décide, ou pas, de voir dans ce singulier un événement morphosémantique. D’une certaine façon, on rejoint ici les remarques plus générales de S. Vinolo portant sur la ligne de partage séparant, en principe, « les événements des faits non-événementiels » $(2013: 52)$ :

[...] Imaginons que nous trouvions un lingot d'or dans la rue. Comment savoir si celui-ci a été perdu ou placé à dessein sur notre chemin par un mécène souhaitant financer nos recherches tout en restant anonyme? C'est nous qui déciderons comment nous recevons ce lingot, et si nous le recevons en tant que don événementiel ou pas. Il en va de même pour tout surgissement phénoménal, pour l'intégralité des phénomènes, pas seulement pour les événements en tant qu'ils se distingueraient des faits. Nous pouvons bouleverser notre vie et notre monde à partir de n'importe quel phénomène qui se donne, en le recevant comme événement. (2013: 64)

Autrement dit, l'événementialité, ici, est une affaire d'herméneutique (Vinolo, $2013: 64$ ), et dépend de la « relation » (Marion, cité par Vinolo, $2013: 64$ ) que le sujet entretient avec le phénomène. C'est bien évidemment, dans l'ordre du style, le principe de motivation et de pertinence interprétative qui fonde, à réception, le sentiment d'un double conditionnement de la forme et du sens. En l'occurrence, il est difficile de ne pas relever le recours au singulier « est » dans le contexte des Pensées de Pascal.

Il va de soi que l'exploration stylistique de la puissance représentationnelle de la grammaire peut concerner, non un morphème grammatical, mais un fait de syntaxe. Ainsi dans cet autre extrait des Pensées :

\section{Perpétuité.}

Qu'on considère que depuis le commencement du monde l'attente ou l'adoration du Messie subsiste sans interruption, qu'il s'est trouvé des hommes qui ont dit que Dieu leur avait révélé qu'il devait naître un Rédempteur qui sauverait son peuple. Qu'Abraham est venu ensuite dire qu'il avait eu révélation qu'il naitrait de lui par un fils qu'il aurait, que Jacob a déclaré que de ses douze enfants il naîtrait de Juda, que Moïse et les prophètes sont venus ensuite déclarer le temps et la manière de sa venue, qu'ils ont dit que la loi qu'ils avaient n'était qu'en attendant celle du Messie, que jusque-là elle serait perpétuelle, mais que l'autre durerait éternellement. Qu'ainsi leur loi ou celle du Messie dont elle était la promesse serait toujours sur la terre, qu'en effet elle a toujours duré, qu'enfin est venu Jésus-Christ dans toutes les circonstances prédites. Cela est admirable. (Pensées, éd. P. Sellier, Paris, Classiques Garnier, 2011, p. 160-161)

Ce qui est "admirable ", dans l'ordre de l'écriture, c'est, à la fin du fragment ${ }^{13}$, la postposition du sujet «Jésus-Christ» dans la subordonnée "qu'enfin est venu JésusChrist dans toutes les circonstances prédites ». Hapax distributionnel, il est préparé en amont par la construction unipersonnelle annonçant la naissance du Sauveur « il devait naître un Rédempteur qui sauverait son peuple ", où le sujet $i l$, marque de la personne d'univers, est comme en attente de spécification et où la séquence est naturellement placée après le verbe. La postposition du « vrai » sujet syntaxique « Jésus-Christ » prend 
localement valeur d'événement - c'est un écart immanent à la norme individuelle du texte.

Existe-t-il des événements négatifs, qui ne sont tels que parce que le contexte fait attendre quelque chose qui ne se produit pas? Assurément, la forme de «présence » de l'événement peut être un manque, une absence, une attente non réalisée. Nous avons par exemple relevé (voir Laurent, 2013) la rareté du passage du pronom vous au pronom tu dans la Bérénice de Racine. Cette énallage se produit deux fois entre Titus et Bérénice, mais jamais dans une situation d'énonciation actuelle : elle est liée à une scène imaginée, ou fictive. L'intrigue racinienne ressortit constamment au domaine public, et le sujet de Bérénice est donc l'impossibilité d'une parole privée: le pronom tu, marque de la transgression, est du côté de l'indicible, l'attouchement étant interdit dans l'univers du "vous », qui incarne la loi. Cette absence d'un tu «actuel» se présente pour ainsi dire réflexivement - au point de concentrer la poétique de la pièce : c'est un événement créé par les interrelations du contexte. À tout le moins, s'il n'est pas nécessairement constaté par la pensée consciente et s'il n'est donc pas nécessairement reçu comme événement spécifique, il est perçu car participant à l'effet global du texte.

\section{Désénoncer l'événement}

31 De quelle «pragmatique du style » est-il question ici? La pragmatique du style n'a pas nécessairement à être analysée, dans son principe, en termes énonciatifs : ce n'est pas parce qu'un style ou un fait de style ont un effet sur le récepteur que leur traitement relève forcément d'une perspective énonciative. Indépendamment des effets rhétoriques de surface, la pragmatique de ce que nous nommons ici "style» élargit dans la communication même le face-à-face de la langue et de l'univers qui est à la source des mécanismes de représentation. Nous ne pouvons qu'être d'accord avec J. Gardes-Tamine quand celle-ci estime que

dans l'écrit, il faut déplacer l'attention de la fonction de communication, première dans l'oral, vers une autre fonction, celle d'exprimer la pensée. (2005: 33)

G. Guillaume valorise un paradigme représentationnel, et non communicationnel, lors de l'examen de la « source » du langage :

le langage est un fait social. On n'en saurait douter : c'est évident. [...] Le langage permet aux hommes en qui, sous la forme stable de langue, il s'est institué durablement, de se communiquer les uns aux autres des idées et des sentiments de tous ordres. Mais sous sa forme humaine de discours adossé à une langue, le langage ne relève-t-il que de cette seule visée ? Et n'est-ce pas le diminuer et en méconnaître l'essence que de n'y voir qu'un certain mode optimum du rapport mutuel des hommes ? Est-ce bien là, dans ce rapport intermittent, que le langage se crée, prend forme? [...] Le langage intervient utilement, à la condition qu'il leur soit - par idéation notionnelle et par idéation structurale - commun, entre des hommes ayant à se dire des choses ayant trait, non pas expressément à leur rapport dans la société à laquelle ils appartiennent, mais à un rapport d'une tout autre espèce, qui est celui de tous et chacun à l'univers, lieu de leur existence. C'est de ce rapport seulement, substrat de tous les autres, y compris le rapport social direct, que les hommes peuvent s'entretenir. [...] Le langage humain a son départ - et des philosophes clairvoyants en ont eu l'aperception - non pas au petit face-à-face Homme / Homme, mais au grand face-à-face Univers / Homme. Là est sa source, et la structure de la langue en porte d'irrécusables témoignages. (2003:129) 

une connaissance de cet univers, mais à la possession d'une lucidité qui permette de le connaître » (2003: 17), le style lui-même présente en quelque sorte une théorie singulière du réel (voir Laurent, 2013) : il engage les conditions mêmes d'une activité de représentation qui fonde, en dernier ressort, les mécanismes constructeurs de la langue. C'est à la langue - comme le montre L. Jenny - que s'adresse l'invention du style, mouvement par lequel la dicibilité d'un non-verbal est comme posée, pesée, ou conquise. Un discours aussi rhétoriquement déterminé qu'un sermon de Bossuet possède une «force» représentationnelle qui ne peut s'expliquer uniquement par le croisement de propriétés extrinsèques. On peut faire les mêmes remarques au sujet du en de Pascal ou de la flexion au singulier du verbe être dans les Pensées. L'absence d'un tu réellement adressé à l'autre par Titus ou Bérénice prend place dans la communication établie entre les deux personnages, mais c'est un événement stylistique en tant qu'il intervient crucialement dans la forme générale et donc dans le sens de l'œuvre - ce qui revient d'ailleurs, chez Racine, à transformer les contraintes de l'art dramatique en véritables choix stylistiques, puisque le sujet de la pièce n'est pas autre chose que l'impossibilité d'une parole qui ne soit pas soumise à la loi de la représentation. Ce qu'on veut dire ici, ce n'est pas que le style ne communique pas - c'est bien un «fait social» - mais que dans son principe, ce qui fait que c'est un style et pas autre chose (par exemple une somme de procédés ou une rhétorique), c'est un processus singulier où se rejouent les conditions mêmes d'une activité de représentation.

Défini ainsi, le style détermine bel et bien une dynamique de figuration et sa puissance esthétique est inséparable d'une dimension cognitive. N. Goodman a raison de noter que le plaisir esthétique se situe cognitivement dans la découverte de relations sémiotiques. La pragmatique du style est là, et se révèle dans le fait que «nous concentr[i]ons notre attention sur les frontières » $(1990: 302)$ - sur les formes du sens.

L'événementialité du style, on l'a vu, repose sur un ensemble de processus où se combinent, en un système complexe, soulignement ou invention de la forme - ce que Gumbrecht appelle l'« effet de présence » et qu'on peut voir comme un processus réflexif par lequel la forme se signale comme telle, fût-ce, dans le cas du tu de Bérénice, négativement - et " effets de signification ». L'individuation discursive est fonction d'une densification «monadique » de l'œuvre, dans la mesure où, d'un certain point de vue, le style "est son propre fond", pour reprendre l'expression de S. Chauvier (2010:4). Cet approfondissement « descendant » (Schaeffer, 2011:145 ${ }^{14}$ ) de l'œuvre, qui découvre des structurations sémiotiques multiples, est toutefois, évidemment, contextualisé et le pouvoir créateur est donc d'autant plus sensible que, d'un autre côté, la dimension de "hénade » de l'œuvre est (ré-)affirmée. Dans cet approfondissement, il ne faut pas oblitérer l'importance objective des mots grammaticaux et des faits de syntaxe par lesquels se noue, dans l'événementialité du style, quelque chose qui ressemble à une « vérité artistique ». 


\section{BIBLIOGRAPHY}

Badiou, Alain (2006) : Logiques des mondes. L'être et l'événement, 2, Paris, Seuil.

- (2009) : Second manifeste pour la philosophie, Paris, Fayard.

- (2018) : L'Immanence des vérités. L'être et l'événement, 3, Paris, Fayard.

Badiou-Monferran, Claire \& Ducos, Joëlle dirs (2012) : « L'émergence : un concept opératoire pour les sciences du langage?", L'Information grammaticale, $\mathrm{n}^{\circ} 134$.

Cadiot, Pierre (1997) : Les Prépositions abstraites en français, Paris, Colin.

Chauvier, Stéphane (2010a) : Le sens du possible, Paris, Vrin.

- (2010b) : «L'unique en son genre », Philosophie, n 106, p. 3-22.

Fagot-Largeault, Anne (2002) : « L'émergence », in Andler, D., Fagot-Largeault, A. \& Saint-Sernin B., Philosophie des sciences II, Paris, Gallimard, coll. « Folio », p. 939-1048.

Fournier, Nathalie (1998) : Grammaire du français classique, Paris, Belin.

Gardes-Tamine, Joëlle (2005) : Pour une grammaire de l'écrit, Paris, Belin.

Goodman, Nelson (1990) : Langages de l'art. Une approche de la théorie des symboles, Paris, Hachette (trad. de Languages of Art: An Approach to a Theory of Symbols, 1976).

Granger, Gilles Gaston (1988 [11968]) : Essai d'une philosophie du style, Paris, Odile Jacob.

Guillaume, Gustave (2003) : Prolégomènes à la linguistique structurale I, Presses de l'Université Laval. Gumbrecht, Hans Ulrich (2010) : Éloge de la présence. Ce qui échappe à la signification, Paris, Libella Maren Sell Editions (trad. de Production of Presence. What meaning cannot convey, Stanford University Press, 2004).

Herschberg-Pierrot, Anne (2005) : Le Style en mouvement. Littérature et art, Paris, Belin.

Jenny, Laurent (1990) : La Parole singulière, Paris, Belin.

- (2000) : «Du style comme pratique », Littérature, $\mathrm{n}^{\circ}$ 118, p. 98-117.

- (2011) : «Introduction », in Jenny, L. dir., Le Style en acte. Vers une pragmatique du style, Genève, MētisPresses, p. 11-15.

Kleiber, Georges (1999) : Problèmes de sémantique. La Polysémie en questions, Villeneuve d'Ascq, P. U. du Septentrion.

Laurent, Nicolas (2013) : « Du discours à l'œuvre », in Badiou-Monferran, C. dir., La Littérarité des belles-lettres. Un défi pour les sciences du texte ?, Paris, Classiques Garnier, p. 197-211.

Macé, Marielle (2010) : « Extension du domaine du style », Critique, 1-2, n 752-753, p. 3-5.

Martin, Robert (1994) : « Préliminaire », in Molinié, G. \& Cahné, P. dirs, Qu'est-ce que le style ?, Paris, PUF, p. 9-13.

Mazaleyrat, Jean \& Molinié, Georges (1989) : Vocabulaire de la stylistique, Paris, PUF.

Morin, Edgar (1977) : La Méthode, 1. La Nature de la nature, Paris, Seuil. 
Schaeffer, Jean-Marie (2011) : « Styles attentionnels et relation esthétique », in Jenny, L. dir., Le Style en acte. Vers une pragmatique du style, Genève, MētisPresses, p. 139-149.

Vigier, Denis (2017) : «L'évolution des usages des prépositions en, dans, dedans entre le XVI et le $\mathrm{XX}^{\mathrm{e}}$ siècle : approche distributionnelle sur corpus outillé », Discours [en ligne], $\mathrm{n}^{\circ} 21$.

Vinolo, Stéphane (2013) : «L'apostrophe de l'événement. Romano à la lumière de Badiou et Marion ", Journal of French and Francophone Philosophy - Revue de la Philosophie française et de langue française, XXI, n², p. 51-67.

Yocaris, Ilias (2016) : Style et semiosis littéraire, Paris, Classiques Garnier.

\section{NOTES}

1. R. Martin évoque l'existence de concepts régulateurs, mais il paraît plus juste de dire qu'il existe des usages régulateurs et non régulateurs d'un même concept, et qu'il existe donc des emplois régulateurs et non régulateurs, réflexifs, du concept de style.

2. Les « relations latérales " peuvent être « intratextuelles » ou bien plus « contextu[elles] », liées alors à des "choix de lecture" spécifiques et engageant "un rapport du type [texte-inter/ paratexte] ou bien [texte-entour pragmatique]» (Yocaris, $2016: 41-42$ ).

3. Voir aussi, sur la possibilité d'appliquer (ou non) le concept d'émergence aux sciences du langage, le numéro 134 de L'Information grammaticale coordonné par C. Badiou- Monferran et J. Ducos (2012).

4. Voir aussi la synthèse proposée par A. Fagot-Largeault (2002).

5. Autrement dit, si l'œuvre en tant qu'événement n'est pas entièrement déductible de ce que l'on sait du monde pré-événementiel (et donc de son contexte historique), elle possède une « réalité propre » qui explique pour partie son événementialité, et cette "réalité » invite, au contraire, à un point de vue « continuiste » sur le style. Ce point de vue, d'ailleurs, n'implique pas que tous les faits de style soient co-orientés, mais que les interrelations stylistiques soient simplement sémantiquement motivées.

6. Nous posons donc que l'événementialité du style consiste en un ensemble complexe d'émergences.

7. Sur le rapport existant entre pensée conceptuelle et pensée du possible, voir Chauvier, 2010a, 85-86.

8. Ce en quoi une approche «monadique » du style contient toujours, à un certain degré, une dimension « hénadique ».

9. Nous soulignons.

10. Sur en et dans, voir notamment Cadiot, 1997: 197-198. Pour une mise en perspective diachronique, voir notamment Vigier, 2017.

11. On rappelle que l'antéisagoge «consiste en ce que le locuteur présente une réalité négativement, pour mieux faire ressortir l'affirmation d'une situation opposée » (Molinié et Mazaleyrat, $1989: 20)$.

12. Voir http://www.penseesdepascal.fr/I/I22-moderne.php.

13. La résolution de cette attente précède une anaphore résomptive opérée par le pronom démonstratif cela qui, comme le souligne l'un des deux relecteurs, que je remercie, "met manifestement en relief dans ce contexte l'unité profonde et insécable d'un monde gouverné dans son intégralité par la Providence divine ».

14. J.-M. Schaeffer oppose le "traitement ascendant de l'information» aux "traitements descendants attentionnellement guidés », qui tendent vers «une dé-hiérarchisation » (2011: 145). 


\section{ABSTRACTS}

Le style, en lequel il convient de ne pas voir une entité statique mais quelque chose qui se constitue en acte, dans l'œuvre, gagne à être envisagé dans sa relation avec la notion d'événement. S'interroger sur le style, dans ce cadre, invite à considérer l'événementialité des formes, leur manière d'apparaître et de signifier en contexte. On montre que la notion d'émergence, entendue ici lato sensu, permet de modéliser des interrelations complexes dont le style est le lieu, ou le lien, invitant, à réception, à parcourir l'entier du continuum discursif. Le point de vue développé ici est celui d'un ancrage référentiel du style, d'une mise en tension, à travers lui, de la langue et du «monde », formant un événement de représentation. Au cœur de cette événementialité des formes et des significations, il faut placer les traits et les morphèmes grammaticaux, éléments essentiels du style et de sa dynamique représentationnelle.

\section{INDEX}

Mots-clés: style, événement, émergence, individuation, grammaire

\section{AUTHOR}

\section{NICOLAS LAURENT}

ENS de Lyon /IHRIM (UMR 5317)

Nicolas Laurent est maître de conférences en linguistique et stylistique françaises à l'École Normale Supérieure de Lyon et membre de l'IHRIM (UMR 5317). Ses travaux portent en particulier sur le nom propre et ses « seuils » (La Part réelle du langage. Essai sur le système du nom propre et sur l'antonomase de nom commun, Paris, Champion, 2016), la pensée de l'individu dans la langue, la grammaire de la phrase, la sémantique et la stylistique des mots grammaticaux, l'épistémologie du style. 